\title{
An EEG-based real-time cortical rhythmic activity monitoring system
}

\author{
Chang-Hwan Im ${ }^{1}$, Han-Jeong Hwang ${ }^{1}$, Huije Che ${ }^{1}$ and Seunghwan Lee ${ }^{2}$ \\ ${ }^{1}$ Department of Biomedical Engineering, Yonsei University, Wonju, 220-710 Korea \\ 2 Department of Neuropsychiatry, Inje University Ilsan-Paik Hospital, Koyang-city, Korea \\ E-mail: ich@yonsei.ac.kr
}

Received 26 February 2007, accepted for publication 5 July 2007

Published 5 September 2007

Online at stacks.iop.org/PM/28/1101

\begin{abstract}
In the present study, we introduce an electroencephalography (EEG)-based, real-time, cortical rhythmic activity monitoring system which can monitor spatiotemporal changes of cortical rhythmic activity on a subject's cortical surface, not on the subject's scalp surface, with a high temporal resolution. In the monitoring system, a frequency domain inverse operator is preliminarily constructed, considering the subject's anatomical information and sensor configurations, and then the spectral current power at each cortical vertex is calculated for the Fourier transforms of successive sections of continuous data, when a particular frequency band is given. A preliminary offline simulation study using four sets of artifact-free, eye-closed, resting EEG data acquired from two dementia patients and two normal subjects demonstrates that spatiotemporal changes of cortical rhythmic activity can be monitored at the cortical level with a maximal delay time of about $200 \mathrm{~ms}$, when 18 channel EEG data are analyzed under a Pentium4 $3.4 \mathrm{GHz}$ environment. The first pilot system is applied to two human experiments-(1) cortical alpha rhythm changes induced by opening and closing eyes and (2) cortical mu rhythm changes originated from the arm movements - and demonstrated the feasibility of the developed system.
\end{abstract}

Keywords: EEG, cortical rhythmic activity, source imaging, inverse problem (Some figures in this article are in colour only in the electronic version)

\section{Introduction}

Cortical rhythmic activity, which is often called spontaneous brain activity or oscillatory brain activity, is generated intrinsically rather than as phase-locked responses to external stimuli 
(Jensen and Vanni 2002). In electroencephalography (EEG) and magnetoencephalography (MEG), the first recorded signal was the alpha rhythm, which is a kind of cortical oscillation peaking at about $10 \mathrm{~Hz}$.

Recently, an increasing number of neuroscientists are becoming interested in cortical rhythmic activity since various in vivo studies in both humans and animals have revealed that cortical rhythmic activity at various frequencies might be closely related to information encoding in the brain (Salenius et al 1995, Salmelin and Hari 1994a, Gruber et al 1999, Kaiser et al 2003, Miltner et al 1999, Kwon et al 1999, Osipova et al 2005). For instance, cortical rhythmic activity might reflect specific body movements and behavioral states. The alpha rhythm peaking at around $10 \mathrm{~Hz}$ becomes strongest when the subject has his eyes closed and is suppressed when the subject is exposed to visual stimuli (Salenius et al 1995). The mu rhythm, with both $10 \mathrm{~Hz}$ and $20 \mathrm{~Hz}$ components, is dampened by limb movements or tactile stimulations (Salmelin and Hari 1994a). It has also been revealed by numerous studies (Gruber et al 1999, Kaiser et al 2003, Miltner et al 1999) that gamma-band activity (30-100 Hz) can be modulated by various behavioral states such as attention, working memory and associative memory. Moreover, changes of cortical rhythmic activity are believed to be involved in various brain diseases such as schizophrenia (Kwon et al 1999) and Alzheimer's disease (Osipova et al 2005).

EEG and MEG are excellent tools to investigate human cortical rhythmic activity noninvasively thanks to their superior temporal resolutions to other noninvasive brain mapping techniques such as functional magnetic resonance imaging (fMRI), positron emission tomography (PET), near infrared spectroscopy (NIRS) and so on. Many studies have been performed to evaluate the coherence between signals acquired at different scalp EEG electrodes or MEG sensors, and investigate spatial signal power patterns appearing in the scalp potential maps or magnetic field maps on the sensor plane (Jensen and Vanni 2002, Salenius et al 1995, Salmelin and Hari 1994a, Gruber et al 1999, Kaiser et al 2003, Miltner et al 1999, Kwon et al 1999, Osipova et al 2005, Kubler et al 2001). However, the EEG or MEG topographies cannot be directly attributed to the underlying cortical regions since sensors may contain information from multiple brain sources, some of which might overlap, and the topographic maps might be smeared out due to the inhomogeneous conductivity distributions in the human head. A deep tangential source might generate two distinct peaks on the topographic map, which are hard to distinguish from two radial sources around the peak locations. Moreover, a very small cortical activation in some cortical areas could yield widespread field distribution in the topographic maps, preventing one from identifying the correct location of the actual cortical source and investigating the coherence between different sensors. If a subject's head is tilted, especially in a helmet-type MEG, so that one hemisphere is closer to the sensors than the other is, one could observe stronger activity at sensors closer to the subject's head even when the strengths are equal at the cortical level. Therefore, to overcome these limitations, source imaging of rhythmic activity at the cortical level is necessary.

Over the last decade, several methods for source imaging of cortical rhythmic activity have been proposed, such as sequential dipole modeling (Congedo et al 2004), dynamic imaging of coherent sources (DICS) (Salmelin et al 2000), frequency-domain minimum current estimation (FD-MCE) (Gross et al 2001), synthetic aperture magnetometry (SAM) (Singh et al 2002) and spectral spatiotemporal imaging (Lin et al 2004). Despite the recent progress in the imaging techniques, source imaging of cortical rhythmic activity has rarely been applied to the real-time brain activation monitoring system. Congedo et al $(2004,2006)$ attempted to apply inverse solutions to the EEG neurofeedback system for the first time. They applied low-resolution electromagnetic tomography (LORETA) software (http://www.unizh.ch/keyinst) to the EEG data which were bandpass-filtered for a specific frequency band. Their system enhanced the 
efficiency of conventional neurofeedback systems which relied only upon the EEG or MEG topographic maps, by tracking spectral power changes at a region of interest (ROI) in a standard human brain. Their approach is meaningful enough in that they first implemented a real-time cortical rhythmic activity monitoring system. Basically, however, the conventional approach that used LORETA-key software resulted in too widespread low-resolution images and did not visualize $3 \mathrm{D}$ cortical activation changes in real time. Moreover, they did not concern themselves about the delay time seriously because their application could be implemented without a very high temporal resolution.

The main goal of the present study is to realize a real-time cortical rhythmic activity monitoring system which visualizes instantaneous cortical activation images and is generally applicable to a variety of potential applications such as brain computer interface (BCI), neurofeedback, real-time diagnosis of brain diseases, and so on, with just slight modifications in the operating software. Before implementing a pilot system, offline analysis software which simulates the real-time cortical activation monitoring system is implemented and applied to four sets of artifact-free, eyes-closed, resting EEG data acquired from two dementia patients and two normal male subjects, in order to investigate if the real time cortical activity monitoring system is possible. After confirming the possibility of the system, we implement a first online pilot system which is integrated with a commercial EEG recording device and apply it to two well-known experiments-(1) cortical alpha rhythm changes induced by opening and closing eyes and (2) cortical mu rhythm changes originated from the arm movements - which demonstrate the validity of the real-time cortical activity monitoring system.

The paper is organized as follows. Section 2 introduces the fundamental ideas of the real-time cortical rhythmic activity monitoring system, methods for forward calculation and inverse estimation, and detailed processes of real-time source imaging and visualization. In section 3, we performed off-line tests of the suggested real-time cortical rhythmic activity monitoring system with prerecorded EEG data, in order to investigate if such a system is possible. Some test results of the first pilot system are then presented in section 4. This paper ends with a conclusion which also introduces possible applications of the real-time monitoring system (section 5).

\section{Methods}

\subsection{Concept of a real-time cortical rhythmic activity monitoring system}

The suggested real-time cortical rhythmic activity monitoring system consists of three parts: (1) data acquisition; (2) pre-processing; (3) processing and visualization. Figure 1 shows a schematic diagram to elucidate the concepts of the suggested system. In the data acquisition part, a subject's structural magnetic resonance imaging (sMRI) data are acquired prior to the EEG data recording. In the present simulation and pilot studies, however, MNI standard brain (http://www.mrc-cbu.cam.ac.uk/Imaging/Common/mnispace.shtml\#evans_proc) was utilized instead of the subject's sMRI, since the individual subject's sMRI data were not available. After multi-channel EEG electrodes are attached on the subject's scalp, the relative locations of the electrodes and important anatomical landmarks are measured using a 3D digitizer system. The sMRI data and electrode configurations are then transferred to the pre-processing part.

The pre-processing part plays a role in constructing an inverse operator in which the subject's anatomical information is reflected. Once the linear inverse operator is constructed and saved to a data-storage unit, spatiotemporal changes of cortical rhythmic activities can be monitored in real time by means of a unified processing and visualization part. The processing and visualization part is composed of three independent programs-the FFT program, 


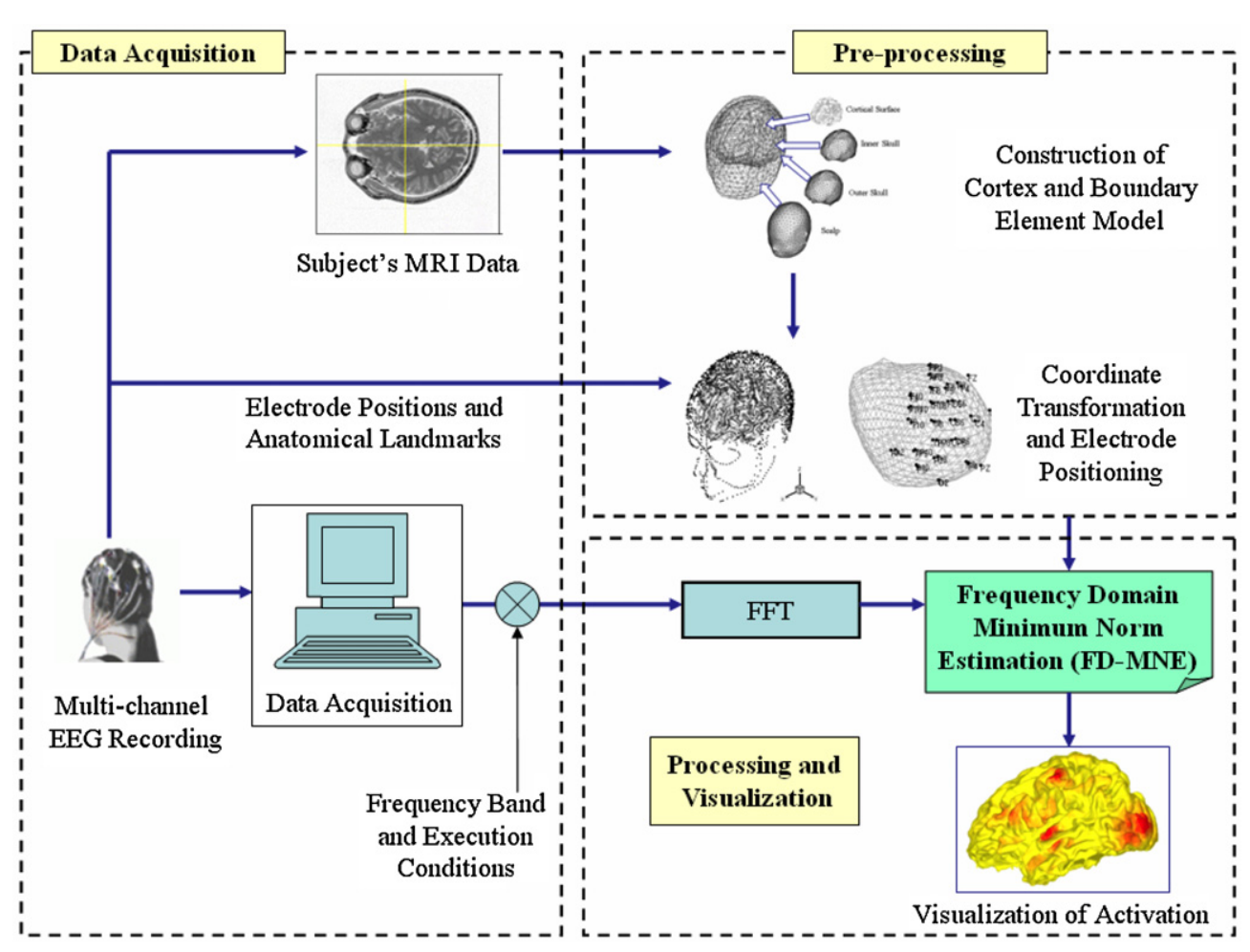

Figure 1. Schematic diagram to elucidate the concepts of the real-time cortical activation monitoring system.

the frequency domain minimum norm estimation (FD-MNE) solver and the visualization program—which are executed one after the other at each time slice.

\subsection{Forward calculation and inverse estimation}

In the present system, a realistic geometry head model was used for accurate EEG forward calculation (He et al 1987, Hämäläinen and Sarvas 1989). A first-order node-based boundary element method (BEM) was applied to construct a lead field matrix which relates source locations to scalp electrodes. In the present study, three-layer tessellated boundary surfaces, consisting of inner and outer skull boundaries and scalp surface, were generated using CURRY5 for windows (Compumedics, Inc., El Paso, TX) from structural MRI data. As stated in the previous section, for all studies, MNI standard brain was utilized. The relative conductivity values of the brain, skull and scalp were assumed to be 1, 1/16 and 1, respectively (Haueisen et al 1997, Oostendorp et al 2000). Coordinate transformation and electrode positioning were performed using in-house software 'BioEST', developed in the Bioelectromagnetics and Neuroimaging Laboratory of Yonsei University (http://bem.yonsei.ac.kr).

Since synchronously activated pyramidal cortical neurons, which are located perpendicularly on the cortical surface, are widely believed to be the main EEG and MEG generators, many recent studies have adopted this physiological phenomenon as a basic anatomical constraint in EEG or MEG source imaging (Dale and Sereno 1993, Kincses et al 


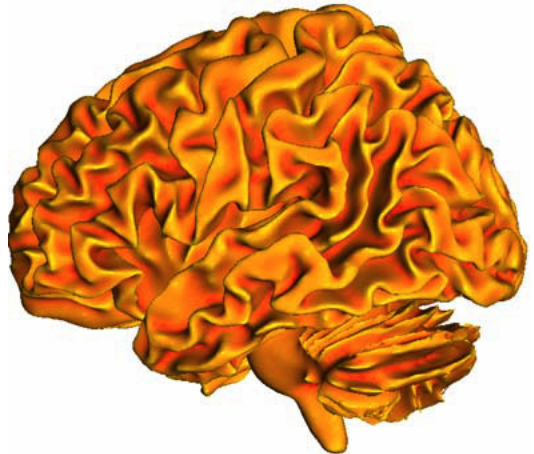

(a)

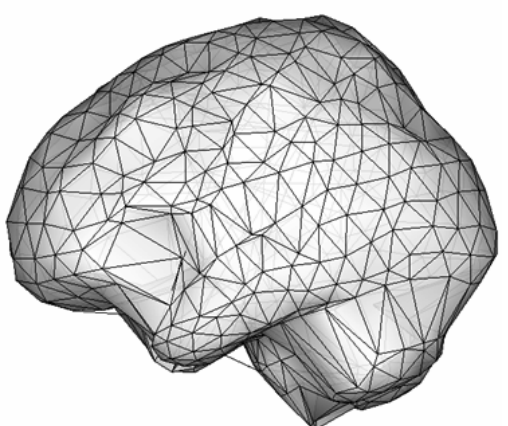

(c)

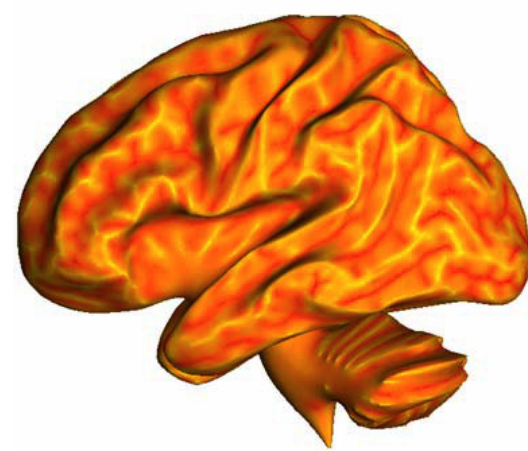

(b)

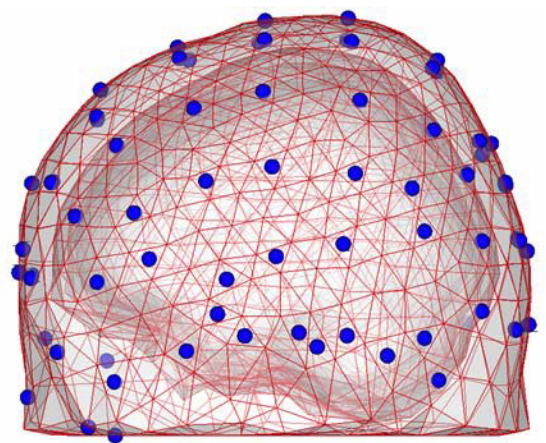

(d)

Figure 2. Generation of cortical source space and boundary element model: (a) original tessellated cortical surface; (b) inflated cortical surface; (c) down-sampled cortical surface model; (d) boundary element model with 99 electrodes. The colors in (a) and (b) represent the distribution of sulci and gyri.

1999, Dale et al 2000, Babiloni 2001). The source imaging with such an anatomical constraint, which has often been called cortically distributed source model or cortical source imaging, resulted in the elimination of spurious sources (Baillet et al 2001) as well as the reduction of crosstalk distribution (Liu et al 1998), compared to conventional volume based imaging techniques.

To impose the anatomical constraint, many dipolar sources are placed on the cortical surface extracted and tessellated from structural MRI data. Although development of medical image processing and high resolution structural MRI enabled us to obtain a high resolution cortical surface with sub-millimeter modeling errors (Dale et al 1999), it is computationally inefficient to use whole cortical surface vertices for the source reconstruction purpose because of the increased underdetermined relationship between limited numbers of sensors and larger numbers of source locations. Therefore, to reduce the number of possible cortical source locations, we first inflated the cortical surface (Fischl et al 1999) and generated a downsampled epi-cortical surface. For the extraction and tessellation of the cortical surface models, we applied BrainSuite developed in the University of Southern California, CA, USA (Shattuck and Leahy 2002). In the present study, about 1000 vertices were down-sampled from more than 400000 original cortical vertices. Figure 2 shows the processes for generating the cortical source space from standard brain MRI data. Figures 2(a) and (b) show the original and inflated cortical surfaces, respectively. Figure 2(c) shows the down-sampled cortical surface on which equivalent dipole sources are placed and figure 2(d) shows the complete boundary element 
models on which 99 electrodes are attached. Since we used the inflated cortical surface model as the source space, the source orientation constraint was not imposed.

To reconstruct the cortically distributed brain sources, we used a linear estimation approach (Dale and Sereno 1993, Lin et al 2004). The expression for the inverse operator W is

$$
\mathbf{W}=\mathbf{R A}^{\mathrm{T}}\left(\mathbf{A R A}^{\mathrm{T}}+\lambda^{2} \mathbf{C}\right)^{-1},
$$

where $\mathbf{A}$ is a lead field matrix which represents the impulse response of each source vector component at every measurement site (Baillet et al 2001), $\mathbf{R}$ is a source covariance matrix representing the inter-source relationship, which is hardly estimated without using intracranial recordings and $\mathbf{C}$ is a noise covariance matrix. If we assume that both $\mathbf{R}$ and $\mathbf{C}$ are scalar multiples of the identity matrix, this approach becomes identical to minimum norm estimation (Liu et al 2002). In this study, the source covariance matrix $\mathbf{R}$ was assumed to be a diagonal matrix, which means that we ignored the relationships between neighboring sources. The lead field weightings were imposed on the lead field matrix to compensate for the sensitivity difference according to the source depth (Lin et al 2006). In this study, the background environmental noise acquired before attaching electrodes on the subject's scalp was used to calculate $\mathbf{C}$ (Lin et al 2004). $\lambda^{2}$ is a regularization parameter and was determined systematically using the following equation (Lin et al 2004):

$$
\lambda^{2}=\frac{\operatorname{trace}\left(\mathbf{A R A} \mathbf{A}^{T}\right)}{\operatorname{trace}(\mathbf{C}) \mathrm{SNR}^{2}}
$$

where trace( $\cdot)$ and SNR represent the sum of diagonal terms and the signal-to-noise ratio, respectively. The signal-to-noise ratio could be determined after some preliminary recordings of continuous EEG signals.

\subsection{Processing and visualization}

The processing and visualization part is composed of three independent programs-the FFT program, the frequency domain minimum norm estimation (FD-MNE) solver and the visualization program - which are executed one after the other at each time slice. At a certain time slice, time domain signals in $2^{N}$ data samples before the time slice are transformed into frequency domain signals using a self-executable, in-house FFT program coded based on Netlib library routines (http://www.netlib.org). The number $N$ could be modified by users (e.g. $N=7,8,9$ and 10) according to their purpose of using the monitoring system. In the present study, we used $N=7$ for all simulation and pilot studies. Once a specific frequency band is determined, the FFT program stores real and imaginary components at all discrete frequencies within the predetermined frequency band to an ASCII data file. Then, the FD-MNE solver is executed and load the Fourier transformed signals $\mathbf{B}\left(f_{i}\right)_{\operatorname{Re}}$ and $\mathbf{B}\left(f_{i}\right)_{\operatorname{Im}}, i=1,2, \ldots, n$, where Re and Im represent the real and imaginary parts of the Fourier transformed signals, respectively, as well as the pre-saved inverse operator $\mathbf{W}$. The real part $\mathbf{q}_{j}\left(f_{i}\right)_{\operatorname{Re}}$ and imaginary part $\mathbf{q}_{j}\left(f_{i}\right)_{\text {Im }}$ of the current source vector at the $j$ th cortical vertex with respect to the frequency of interest $f_{i}$ can then be evaluated by multiplying the corresponding rows $(3 j-2,3 j-1$ and $3 j$ th rows) in $\mathbf{W}$ with the Fourier transformed signals $\mathbf{B}\left(f_{i}\right)_{\operatorname{Re}}$ and $\mathbf{B}\left(f_{i}\right)_{\operatorname{Im}}$. Finally, the absolute current source power at the $j$ th cortical vertex with respect to the frequency band of interest is calculated as

$$
\mathbf{Q}_{j}=\frac{1}{2 n} \sum_{i=1}^{n}\left(\left\|q_{j}\left(f_{i}\right)_{\mathrm{Re}}\right\|^{2}+\left\|q_{j}\left(f_{i}\right)_{\mathrm{Im}}\right\|^{2}\right) .
$$


This process is equivalent to the conventional frequency domain minimum current estimates (FD-MCEs) proposed by Jenson and Vanni (Jensen and Vanni 2002). While the conventional FD-MCEs have used L1 norm-based nonlinear optimization, the FD-MNE approach used in the present study is based on L2 norm-based linear optimization. We adopted the FD-MNE approach since L1 norm based optimization requires time-consuming nonlinear iteration, which is inadequate for the real-time monitoring system.

After the current source power at every cortical vertex is calculated, a 3D visualization program is executed and visualizes the resultant source distribution at a given frequency band. The visualization program named MeshViewer, which was coded with visual $\mathrm{C}++$ under an OpenGL environment, can visualize instantaneous and/or averaged source power changes in real-time from any 3D viewpoints.

\section{Possibility of the real-time cortical rhythmic activity monitoring system: a preliminary simulation study}

\subsection{Simulation setups and materials}

For the verification of the suggested real-time cortical activation monitoring system, offline simulations which perfectly simulated the suggested system were conducted. Four sets of artifact-free, eye closed, resting EEG data acquired from two male dementia patients and two normal male subjects were used to show the possibility of the suggested system. The experimental data were acquired in the Department of Neuropsychiatry of Inje University Ilsan-Paik Hospital, Korea. Written informed consent was obtained from the subjects or their close relatives. The control subjects had no history of neurological, psychiatric or other severe diseases. The patients had no history of stroke, head trauma or any other neurological diseases except gradual decline of cognitive functions and memory. The number of electrodes used for the recording was 18 (Fp1, F3, C3, P3, Fp2, F4, C4, P4, F7, T7, P7, O1, F8, T8, P8, $\mathrm{O} 2, \mathrm{~T} 1, \mathrm{~T} 2$ ) and the sampling frequency was $250 \mathrm{~Hz}$. Since the individual subject's sMRI data were not available then, a standard cortex-head model extracted from MNI standard brain atlas was utilized. For more realistic simulations, the $20 \mathrm{~s}$ EEG data were stored preliminarily in a computer memory and were transferred to a signal variable array, one after another at every $4 \mathrm{~ms}$. At a specific time slice, time domain signals in $2^{N}$ data samples before the time slice are transformed into frequency domain signals using the FFT program. We used $N=7$ and updated the cortical activation maps at every $500 \mathrm{~ms}\left(2\right.$ image frames $\left.\mathrm{s}^{-1}\right)$. As described before, the linear inverse operator $\mathbf{W}$ in (1) was preliminarily constructed and stored in the computer. Since we had already constructed the standard cortex-head models, the time required for the construction of the inverse operator was less than $3 \mathrm{~min}$. The simulation study was performed in an Intel ${ }^{\circledR}$ Pentium4-3.4 GHz personal computer system with $1 \mathrm{~GB}$ memory.

\subsection{Results of the simulation study}

Figures 3(a) and 3(b) show the cortical alpha activity ( 8 to $13 \mathrm{~Hz}$ ) changes in the dementia patients. The figures show cortical activation maps averaged over $20 \mathrm{~s}$ as well as instantaneous screenshots of the cortical activity maps at 1,2 and $3 \mathrm{~s}$. The two patients showed similar activation patterns in both instantaneous and averaged cortical activation maps. Figures 4(a) and 4(b) show the cortical alpha activity changes in the normal subjects, acquired under the same conditions. Both normal subjects showed very typical cortical activations which are located around the occipital lobe (Salmelin and Hari 1994b, Vanni et al 1997), whereas the dementia patients showed additional strong cortical activations around the right temporal and 


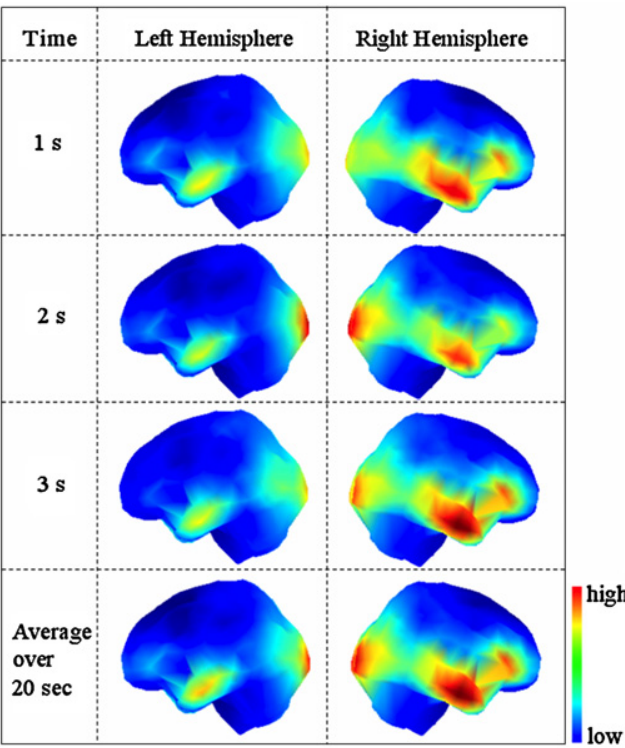

(a)

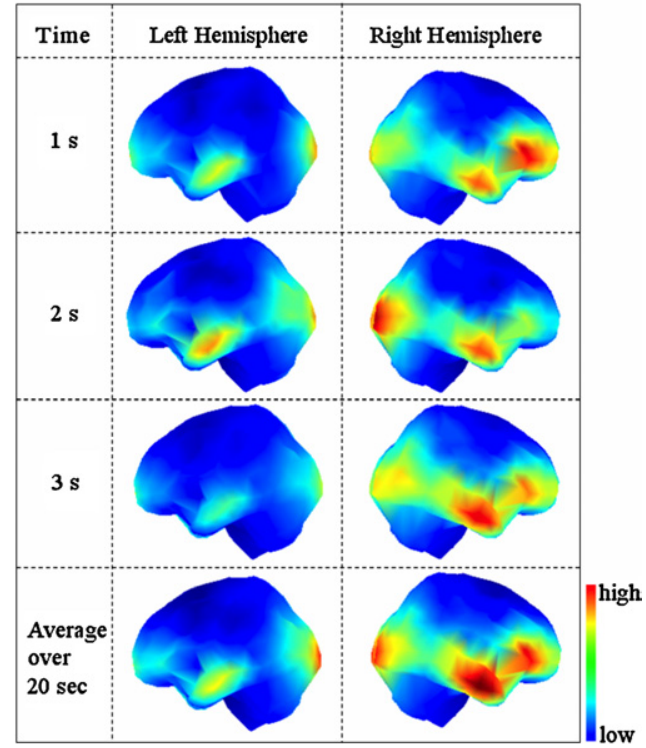

(b)

Figure 3. Cortical alpha $(8-13 \mathrm{~Hz})$ activity changes of dementia patients during a resting EEG recording: (a) dementia patient \#1; (b) dementia patient \#2.

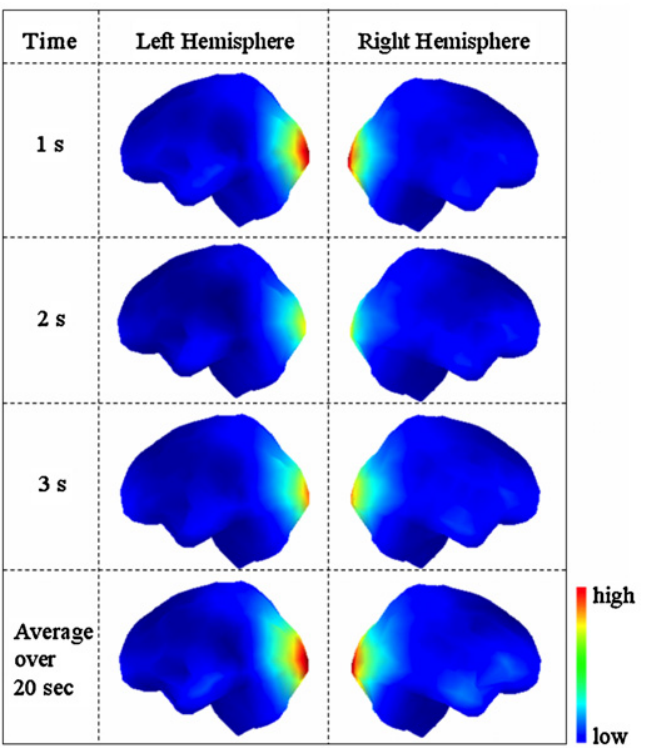

(a)

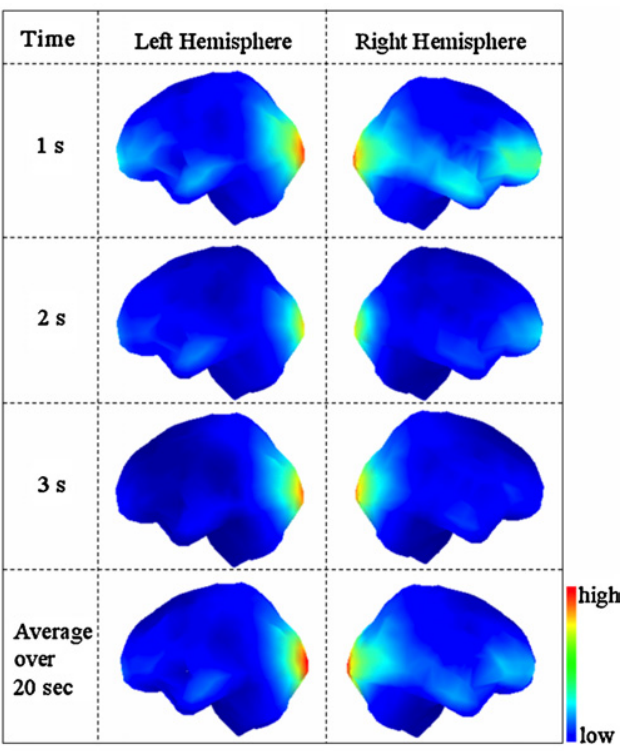

(b)

Figure 4. Cortical alpha $(8-13 \mathrm{~Hz})$ activity changes of normal subjects during a resting EEG recording: (a) control subject \#1; (b) control subject \#2.

frontal lobes and relatively weak additional activations around the left temporal lobe. Such a clear difference in the cortical activation maps of dementia and normal subjects not only shows that the suggested real-time cortical activation monitoring system is working well but 
also demonstrates that the monitoring system can be applied to real-time diagnosis of dementia patients.

The most important issue in realizing the real-time cortical activation monitoring system is to reduce the delay time. Short delay time implies that one can get more cortical activation images during limited acquisition time. In the present simulation study, therefore, some factors influencing the delay time were investigated.

The simulation study was performed in an Intel ${ }^{\circledR}$ Pentium4-3.4 GHz personal computer system with 1 GB memory. The operating system was Microsoft Windows $\mathrm{XP}^{\circledR}$ and the main program including FFT and FD-MNE was executed in the command consol window. The independent visualization software was executed at the first execution and just refreshed at each image frame without closing or opening. During the test, no applications, including virus vaccine, were executed to reduce the system load. For the measurement of the delay time, an intrinsic Fortran function 'date_and_time()' was used. The FFT program and the main FD-MNE program were executed repeatedly and each execution time was averaged over 100 times. We could obtain minimal delay time when 128 data samples were used and the source distribution was reconstructed at a single frequency. Then, the execution of the FFT program and the FD-MNE program took 43.9 and $94.5 \mathrm{~ms}$, respectively. The maximal computational load was required in the present study when we repeated source imaging with 1024 data samples within the whole frequency band of interest $(0-30 \mathrm{~Hz})$. Then, the execution times for FFT and FD-MNE were measured as 59.5 and $128 \mathrm{~ms}$, respectively. When considering about 8-times bigger data size and 123-times more matrix multiplications, the increment of delay time was not significant. The delay time was monotonically increased by the increment of the number of data samples or the number of frequencies of interest. The intrinsic delay time for each program was originated from the CPU time needed for executing programs and time for loading and saving data files. The average time taken to execute the visualization program was $15 \mathrm{~ms}$. Thus, this analysis points out that cortical rhythmic source changes can be monitored at the cortical level with a maximal delay time of about $200 \mathrm{~ms}$ when 18 channel EEG data are analyzed.

For comparison, we also repeated the source imaging with 1024 data samples within the whole frequency band of interest $(0-30 \mathrm{~Hz})$, for 32-channel EEG data acquired from a different EEG recording system (WEEG-32, Laxtha Inc., Korea). Then, the execution times for FFT and FD-MNE were measured to be 62.7 and $137 \mathrm{~ms}$, respectively, suggesting that the increment of recording channels does not highly affect the overall delay time.

\section{A first pilot system: experimental results}

The first pilot system for the EEG-based real-time cortical rhythmic activity monitoring was implemented at the Bioelectromagnetics and Neuroimaging Laboratory of Yonsei University. The EEG was recorded at 16 electrode locations (Fpz, F3, C3, P3, F4, C4, P4, T7, P7, O1, Cz, T8, P8, O2, T1, T2) using the WEEG-32 EEG acquisition system (Laxtha Inc., Daejoen, Korea). The electrodes were attached on the subject's scalp according to extended 10-20 system without using an electrode cap. The EEG was sampled at $256 \mathrm{~Hz}$ and the low- and high-pass filters were set at 64 and $0.5 \mathrm{~Hz}$ cutoffs, respectively. The recorded EEG signals were transferred to an operating computer in real-time and the values were stored in a two-dimensional array variable. The acquisition program was coded in the laboratory using C++. Since the individual subject's sMRI data were not available then, a standard cortex-head model extracted from MNI standard brain atlas was utilized to construct the inverse operator. At a specific time slice, time domain signals in 128 data samples before the time slice are 

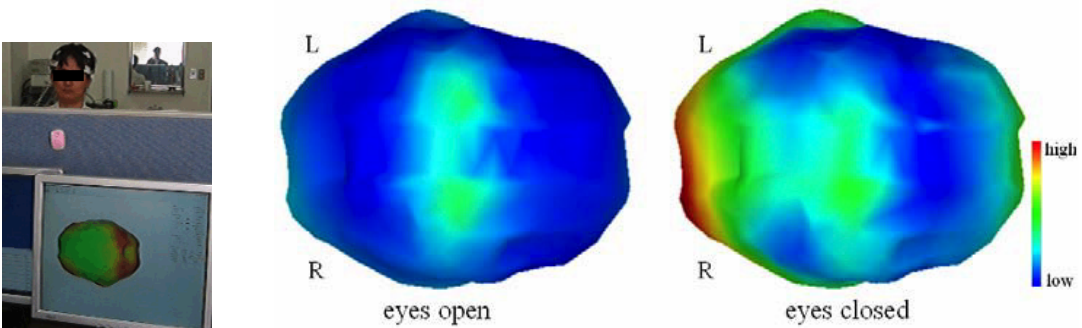

Figure 5. Cortical alpha $(8-13 \mathrm{~Hz})$ activity changes of subject YJ: The left figure shows a photograph of the experiment; the center and right figures show the alpha activity changes when the subject opened and closed his eyes, respectively. Cortical activity maps are viewed from top. ' $L$ ' and ' $R$ ' represent 'left' and 'right', respectively. Values in the source images were normalized to a predetermined threshold which was obtained from some preliminary experiments.
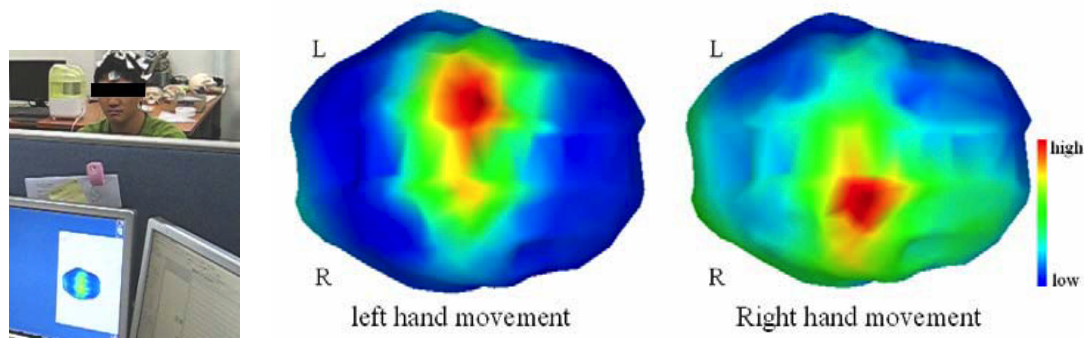

Figure 6. Cortical mu (8-12 Hz) activity changes of subject JJ: The left figure shows a photograph of the experiment; the center and right figures show the mu activity changes when the subject raised his left and right hand, respectively. Cortical activity maps are viewed from top. 'L' and ' $R$ ' represent 'left' and 'right', respectively. Values in the source images were normalized to a predetermined threshold which was obtained from some preliminary experiments.

transformed into frequency domain signals using the FFT program. We updated the cortical activation maps at every $250 \mathrm{~ms}$ (4 image frames $\left.\mathrm{s}^{-1}\right)$.

Two male subjects (YJ and JJ, 26 and 23 years old respectively) volunteered to participate in the test experiments in exchange for monetary compensation. Written informed consent was obtained from the subjects, who had no history of neurological, psychiatric or other severe diseases.

The first subject (YJ) was asked to be relaxed, sitting on a comfortable seat. After the experimental setup is ready, he opened or closed his eyes according to the experimenter's instructions. Figure 5 shows a photograph of the first experiment, where the EEG acquisition system is hidden, as well as two screenshots of the cortical alpha activity ( 8 to $13 \mathrm{~Hz}$ ) changes by the subject's eye closing. When the subject closed his eyes, a gradual increment of cortical activations around the occipital lobe was clearly observed, coinciding well with the results of previous studies (Salmelin and Hari 1994b, Vanni et al 1997).

The other subject (JJ) was asked to slowly raise his right or left arms according to the experimenter's instructions, while opening his eyes. Figure 6 shows a photograph of the second experiment as well as two screenshots of the cortical mu activity $(8-12 \mathrm{~Hz})$ changes by the subject's arm movements. An ipsilateral activity around motor cortex was observed, which is consistent with previous reports (Qin et al 2004, Kamousi et al 2007). The results of both experimental studies coincided well with known physiological phenomena, demonstrating the feasibility of the pilot real-time cortical rhythmic activity monitoring system. 


\section{Conclusions}

In the present study, we implemented a real-time cortical rhythmic activity monitoring system which can monitor spatiotemporal changes of cortical rhythmic activity on a subject's cortical surface with high temporal resolution. In the present system, a frequency domain inverse operator was constructed a priori and the spectral current power at each cortical vertex was then calculated for the Fourier transforms of successive sections of continuous data. An offline simulation study as well as experimental validation studies demonstrated that cortical rhythmic source changes can be monitored at the cortical level with a maximal delay time of about $200 \mathrm{~ms}$.

For the simulation study, we have used four sets of artifact-free, eye closed, resting EEG data acquired from two dementia patients and two normal male subjects to show the possibility of the suggested system. The preliminary offline analysis yielded promising results, suggesting that the real-time cortical activation monitoring system can be potentially used for the real-time diagnosis of psychiatric brain diseases such as dementia and schizophrenia. Application of the 'online' cortical activity monitoring system to the real-time diagnosis of psychiatric brain diseases will be performed in our future studies. For the experimental study, cortical alpha rhythm changes by closing eyes and cortical mu rhythm changes by arm movements were observed, which were consistent with previous reports.

Another possible application of the real-time cortical activation monitoring system is the EEG-based brain computer interface (BCI) system. Although such a system has not been realized yet, some offline simulation studies already demonstrated that the use of inverse solutions could enhance the classification capability of the EEG-based BCI system (Qin et al 2004, Kamousi et al 2005, 2007). As already introduced in the introduction section, the realtime cortical activation monitoring system can be applied to neurofeedback systems in order to enhance the efficiency of detecting the current mental status of a subject. Moreover, the suggested real-time cortical activation monitoring system can be used for online monitoring of EEG experiments regarding various cognitive and functional brain studies. The experimenter can modify the experimental protocols without stopping the on-going measurement with the aid of the suggested system.

\section{Acknowledgments}

This work was supported in part by the Korea Research Foundation Grant funded by the Korean Government (MOEHRD) (KRF-2006-331-D00728) and in part by a grant of the Korea Health 21 R\&D project, Ministry of Health and Welfare, Korea (02-PJ3-PG6-EV07-0002).

\section{References}

Babiloni C 2001 Linear inverse source estimate of combined EEG and MEG data related to voluntary movements Hum. Brain Mapp. 14 197-209

Baillet S, Riera J J, Marin G, Mangin J F, Aubert J and Garnero L 2001 Evaluation of inverse methods and head models for EEG source localization using a human skull phantom Phys. Med. Biol. 46 77-96

Congedo M 2006 Subspace projection filters for real-time brain electromagnetic imaging IEEE Trans. Biomed. Eng. 53 1624-34

Congedo M, Lubar J F and Joffe D 2004 Low-resolution electromagnetic tomography neurofeedback IEEE Trans. Neural Syst. Rehab. Eng. 12 387-97

Dale A M, Fischl B and Sereno M I 1999 Cortical surface-based analysis: I. Segmentation and surface reconstruction Neuroimage 9 179-94 
Dale A M, Liu A K, Fischl B R, Buckner R L, Belliveau J W, Lewine J D and Halgren E 2000 Dynamic statistical parametric mapping: combining fMRI and MEG for high-resolution imaging of cortical activity Neuron 26 55-67

Dale A M and Sereno M I 1993 Improved localization of cortical activity by combining EEG and MEG with MRI surface reconstruction: a linear approach J. Cogn. Neurosci. 5 162-76

Fischl B, Sereno M I and Dale A M 1999 Cortical surface-based analysis: II. Inflation, flattening, and a surface-based coordinate system Neuroimage 9 195-207

Gross J, Kujala J, Hämäläinen M, Timmermann L, Schnitzler A and Salmelin R 2001 Dynamic imaging of coherent sources: studying neural interactions in the human brain Proc. Natl Acad. Sci. USA $98694-9$

Gruber T, Muller M M, Keil A and Elbert T 1999 Selective visual-spatial attention alters induced gamma band responses in the human EEG. Clin. Neurophysiol. $1102074-85$

Hämäläinen M S and Sarvas J 1989 Realistic conductivity geometry model of the human head for interpretation of neuromagnetic data IEEE Trans. Biomed. Eng. 36 165-71

Haueisen J, Ramon C, Eiselt M, Brauer H and Nowak H 1997 Influence of tissue resistivities on neuromagnetic fields and electric potentials studied with a finite element model of the head IEEE Trans. Biomed. Eng. 44 727-35

He B, Musha T, Okamoto Y, Homma S, Nakajima Y and Sato T 1987 Electric dipole tracing in the brain by means of the boundary element method and its accuracy IEEE Trans. Biomed. Eng. 34 406-14

Jensen O and Vanni S 2002 A new method to identify multiple sources of oscillatory activity from magnetoencephalographic data NeuroImage 15 568-74

Kaiser J, Ripper B, Birbaumer N and Lutzenberger W 2003 Dynamics of gamma-band activity in human magnetoencephalogram during auditory pattern working memory NeuroImage 20 816-27

Kamousi B, Amini A N and He B 2007 Classification of motor imagery by means of cortical current density estimation and Von Neumann entropy for brain-computer interface applications J. Neural Eng. 4 17-25

Kamousi B, Liu Z and He B 2005 Classification of motor imagery tasks for brain-computer interface applications by means of two equivalent dipoles analysis IEEE Trans. Neural Syst. Rehabil. Eng. 13 166-71

Kincses W E, Braun C, Kaiser S and Elbert T 1999 Modeling extended sources of event-related potentials using anatomical and physiological constraints Hum. Brain Mapp. 8 182-93

Kubler A, Kotchoubey B, Kaiser J, Wolpaw J R and Birbaumer N 2001 Brain-computer communication: unlocking the locked Psychol. Bull. 127 358-75

Kwon J S, O’Donnell B F, Wallenstein G V, Greene W, Hirayasu Y, Nestor P G, Hasselmo M E, Potts G F, Shenton M E and McCarley R W 1999 Gamma frequency-range abnormalities to auditory stimulation in schizophrenia Arch. Gen. Psychiatry 56 1001-5

Lin F H, Witzel T, Ahlfors S P, Stufflebeam S M, Belliveau J W and Hämäläinen M S 2006 Assessing and improving the spatial accuracy in MEG source localization by depth-weighted minimum-norm estimates Neuroimage 31 160-71

Lin F H, Witzel T, Hämäläinen M S, Dale A M, Belliveau J W and Stufflebeam S M 2004 Spectral spatiotemporal imaging of cortical oscillations and interations in the human brain Neurolmage 23 582-95

Liu A K, Belliveau J W and Dale A M 1998 Spatiotemporal imaging of human brain activity using functional MRI constrained magnetoencephalography data: Monte Carlo simulations Proc. Natl Acad. Sci. USA $958945-50$

Liu A K, Dale A M and Belliveau J W 2002 Monte Carlo simulation studies of EEG and MEG localization accuracy Hum. Brain Mapp. 16 47-62

Miltner W H, Braun C, Arnold M, Witte H and Taub E 1999 Coherence of gamma-band EEG activity as a basis for associative learning Nature 397 434-6

Oostendorp T F, Delbeke J and Stegeman D F 2000 The conductivity of the human skull: results of in vivo and in vitro measurements IEEE Trans. Biomed. Eng. 47 1487-92

Osipova D, Ahveninen J, Jensen O, Ylikoski A and Pekkonen E 2005 Altered generation of spontaneous oscillations in Alzheimer's disease NeuroImage 27 835-41

Qin L, Ding L and He B 2004 Motor imagery classification by means of source analysis for brain-computer interface applications J. Neural Eng. 1 135-41

Salenius S, Kajola M, Thompson W L, Kosslyn S and Hari R 1995 Reactivity of magnetic parieto-occipital alpha rhythm during visual imagery Electroencephalogr. Clin. Neurophysiol. 95 453-62

Salmelin R and Hari R 1994a Spatiotemporal characteristics of sensorimotor neuromagnetic rhythms related to thumb movement Neuroscience $60537-50$

Salmelin R and Hari R 1994b Characterization of spontaneous MEG rhythms in healthy adults Electroencephalogr. Clin. Neurophysiol. 91 237-48

Salmelin R, Schnitzler A, Schmitz F and Freund H J 2000 Single word reading in developmental stutterers and fluent speakers Brain 123 1184-202 
Shattuck D W and Leahy R M 2002 BrainSuite: an automated cortical surface identification tool Med. Image Anal. 6 129-42

Singh K D, Barnes G R, Hillebrand A, Forde E M E and Williams A L 2002 Task-related changes in cortical synchronization are spatially coincident with the hemodynamic response NeuroImage 16 103-14

Vanni S, Revonsuo A and Hari R 1997 Modulation of the parieto-occipital alpha rhythm during object detection $J$. Neurosci. 17 7141-7 